\title{
Analysis of contact resistance in single-walled carbon nanotube channel and graphene electrodes in a thin film transistor
}

\author{
Jinwook Baek, Travis G. Novak, Houngkyung Kim, Jinsup Lee, Byoungwook Jang, Junseok Lee \\ and Seokwoo Jeon*
}

\begin{abstract}
In this work, we present the experimental investigation on the contact resistance of graphene/single-walled carbon nanotube (SWCNT) junction using transfer length method with the simple equivalent circuit model. We find that $p-n$ like junctions are formed in graphene/SWCNT transistors, and the contact resistance in the junction is observed to be $\sim 494$ and $\sim 617 \mathrm{k} \Omega$ in case of metallic SWCNT (m-SWCNT) and semiconducting SWCNT (s-SWCNT), respectively. In addition, the contact resistance increases from 617 to $2316 \mathrm{k} \Omega$ as $\mathrm{V}_{\mathrm{g}}$ increases from -30 to $-10 \mathrm{~V}$. Through our study, high carrier density induced from doping in both graphene and SWCNT leads to low contact resistance. This development of contact engineering, namely modulation of carrier density in the junction and contact length $\left(\mathrm{L}_{\text {con }}\right)$ scaling shows the potential for all-carbon based electronics.
\end{abstract}

\section{Introduction}

One of the key issues in future electronic devices is making ultra-short channel length $\left(\mathrm{L}_{\mathrm{ch}}\right)$ transistors with high performance in order to increase the density of each device. Therefore, CNTs and graphene are perspective electrical materials for future devices due to their high mobility, high on/off ratio and flexibility, with the sizes down to $<10 \mathrm{~nm}$ [1-4]. Therefore, all-carbon thin-film transistors using CNT or graphene as electrodes and channels are expected to enable the fabrication of flexible, transparent and high performance devices using relatively simple and low-cost techniques [5]. However, the full-potential of those exceptional properties can only be fully realized if a transparent, ohmic contact with negligible contact resistance contact is formed between them.

Contact resistance is an inherent property contributing to the total resistance of a system, which can be attributed to the contacting interfaces of electrical leads and connections (as opposed to the intrinsic resistance). In

\footnotetext{
*Correspondence: jeon39@kaist.ac.kr

Department of Materials Science and Engineering, Korea Advanced Institute of Science and Technology (KAIST), 335 Gwahangno, Yuseong-gu, Daejeon 305-701, Republic of Korea
}

case of metal-CNT and CNT-CNT contact devices, the existence of a Schottky barrier at the contact area induces high contact resistance, limiting important performance parameters such as on-state current and field effect mobility. To improve the performance of CNT devices, a good electrical contact must be developed. As a member of the carbon allotropes, Graphene has emerged as an alternative electrode material for CNT devices due to its flexibility, exceptional electric transport, and potential for the seamless contact with CNT. While there have been several experimental and theoretical studies on the graphene/SWCNT junction properties, relatively few studies have been reported values of contact resistance in graphene/SWCNT junctions [6, 7].

Here, we define electrical contact resistance between graphene and SWCNT junction using the transfer length method (TLM) with a simple equivalent model. We have fabricated aligned arrays of SWCNTs with graphene electrode channel TFTs on $\mathrm{Si} / \mathrm{SiO}_{2}$ substrates, with various $\mathrm{L}_{\mathrm{ch}} \mathrm{s}(4-10 \mu \mathrm{m})$ and $\mathrm{L}_{\text {con }} \mathrm{s}(5-25 \mu \mathrm{m})$. The electrical transport properties indicate that $\mathrm{p}-\mathrm{n}$ like junctions are formed in graphene/SWCNT junctions and exhibit contact resistances of $\sim 494$ and $\sim 617 \mathrm{k} \Omega$ in case of each $\mathrm{m}-\mathrm{SWCNT}$ and s-SWCNT, respectively. In addition, we 
find that contact resistance depends significantly on the carrier density and $\mathrm{L}_{\text {con }}$ even in micrometer regime.

\section{Experiment}

\subsection{Synthesis of graphene on Cu foil}

Single layer graphene was grown on the prepared $25 \mu \mathrm{m}$ thick $\mathrm{Cu}$ foil (Alfa Aesar, 13,382) using chemical vapor deposition (CVD) method. The $\mathrm{Cu}$ foil was annealed in a quart tube at $1000{ }^{\circ} \mathrm{C}$ for 20 min under vacuum at $<10^{-3}$ torr with flowing $\mathrm{H}_{2}(10 \mathrm{sccm})$. Thereafter, a mixed gas of $\mathrm{CH}_{4}$ and $\mathrm{H}_{2}$ was injected for $20 \mathrm{~min}$, and the $\mathrm{Cu}$ foil is rapidly cooled down to room temperature. Then the graphene was transferred on the $\mathrm{Si} / \mathrm{SiO}_{2}$ wafer using PMMA as a supporting polymer layer in order to develop TFTs with graphene electrodes [8]. To fabricate two isolated electrodes (source and drain), the lithography process was performed with $\mathrm{O}_{2}$ plasma treatment.

\subsection{Synthesis of SWCNTs on quartz substrate}

Aligned SWCNTs were grown on the pre-annealed $(10 \mathrm{~h}$, $1000{ }^{\circ} \mathrm{C}$ in air) ST-cut quartz (cutting angle: $42^{\circ} 45^{\prime}$, Hoffman Materials, USA) substrate using CVD method. First, catalyst deposited quartz substrate ( $5 \AA$, deposited by e-beam evaporator) was inserted into a quartz tube and then heated up to $920{ }^{\circ} \mathrm{C}$ under ambient pressure with flowing Ar gas (1000 sccm) for pre-annealing of $1 \mathrm{~h}$. After the pre-annealing, mixture of Ar (50 sccm), H2 (10 sccm) and $\mathrm{CH} 4(150 \mathrm{sccm})$ gas was injected to reaction chamber for $20 \mathrm{~min}$ for SWCNTs growth. The quartz substrate was rapidly cooled down to room temperature under $\mathrm{Ar}$ flow (1000 sccm) followed by the SWCNT growth [9]. As-grown SWCNTs were transferred onto patterned graphene $/ \mathrm{SiO}_{2} / \mathrm{Si}$ substrate by using PMMA [Poly (methyl methacrylate)] as a supporting layer, similar to the graphene transfer method.

\subsection{Fabrication of graphene/SWCNT devices}

A mask aligner system (MIDAS system Co., Ltd.) was used for the patterning of graphene, SWCNTs, and metal pad lines on the $\mathrm{Si} / \mathrm{SiO}_{2}$ substrate in which silicon and silicon dioxide served as back-gate electrode and dielectric, respectively. To isolate each sets of TLM test structures, the pattern was formed by depositing a positive photoresist (AZ5214) and then etching by oxygen plasma. After removing the photoresist with acetone, the samples were annealed in a furnace under vacuum $\left(10^{-4}\right.$ torr) with Ar flow $(500 \mathrm{sccm})$ at $400{ }^{\circ} \mathrm{C}$ for $3 \mathrm{~h}$ to remove PMMA and photoresist residue. The $\mathrm{Cr} / \mathrm{Au}$ $(\sim 3-50 \mathrm{~nm})$ pad electrode was e-beam evaporated on the top of graphene electrode in a vacuum with a pressure of $10^{-7}$ torr.

\subsection{Characterization of graphene/SWCNTs TFTs}

The microstructure of the graphene/SWCNT TFTs were observed with a scanning electron microscope (SEM) (Hitachi S4800) and atomic force microscopy (AFM). We analyzed the electrical characteristics using a simple equivalent circuit model in which we assume diffusive transport in the channel. The quality of graphene/ SWCNT TFTs were characterized by Raman spectroscopy (SENTERRA, Bruker GmbH, $532 \mathrm{~nm}$ ). All electrical properties were measured by a probe station (Keithley 4200-SCS).

\section{Results and discussion}

Figure 1a shows a fabricated graphene/SWCNTs junction TFT on $\mathrm{Si} / \mathrm{SiO}_{2}(100 \mathrm{~nm})$ substrate. The single layer graphene was synthesized on the $\mathrm{Cu}$ foil with a conventional CVD method, as reported in our previous work [9]. The highly aligned SWCNTs were fabricated from a CVD growth process with patterned metal catalyst on quartz substrate. They were then transferred sequentially onto $\mathrm{Si} /$ $\mathrm{SiO}_{2}$ substrates by using PMMA as a supporting layer [8]. Detailed description for the fabrication process is given in Sect. 2. During the CVD growth process, aligned SWCNTs are grown with $300 \mu \mathrm{m}$ length between the patterned catalyst lines. The device layout consists of three separately fabricated parts: the SWCNTs channel, the graphene electrodes and the Au probing pads. The $\mathrm{L}_{\mathrm{ch}}$ of the devices was defined by various spacing of patterned graphene $(2,4,6$, $8,10,12$ and $14 \mu \mathrm{m})$ and overlapping length and width (25 and $100 \mu \mathrm{m}$, respectively) with aligned SWCNTs. The additional geometric information of graphene/SWCNTs device was shown in Additional file 1: Figure S1. To prevent damage of graphene electrodes while electrical performance was measured, the Au probing pads $(\sim 50 \mathrm{~nm})$ were deposited by e-beam evaporator on the top of the graphene electrodes. Figure 1b shows an exhibits SEM image of representative $\mathrm{L}_{\mathrm{ch}} \mathrm{S}$ of aligned SWCNTs with the graphene electrodes and probing pads at each device. In previous reports, it was determined that the diameter of individual SWCNTs is an important factor in the device behavior, a result attributed to the band gap parameter of the SWCNTs [10]. Moreover, it provides the Schottky barriers at the contact region between channel and electrode due to the different energy band level. To separate the influence of resistance induced in contact region, the diameter distribution of aligned SWCNTs on a $\mathrm{SiO}_{2}$ substrate was measured by AFM as shown in Fig. 1d, e. The densities of SWCNTs were $0.8 \pm 0.2$ SWCNTs $\mu \mathrm{m}^{-1}$ and average diameter distribution at $\sim 0.87 \mathrm{~nm}$. Moreover, for the differentiation of the SWCNTs chirality in this sample, AFM images were used with Raman spectra of SWCNTs to measure the diameter distribution. Consequently, 

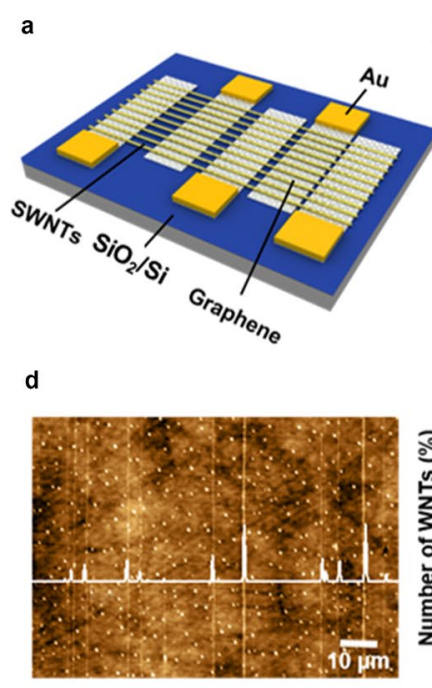
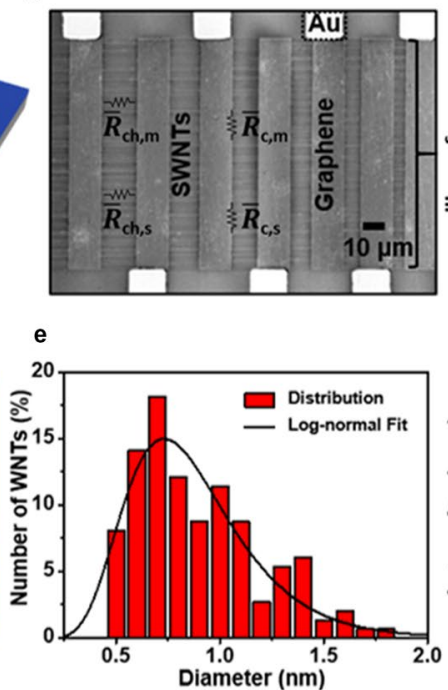
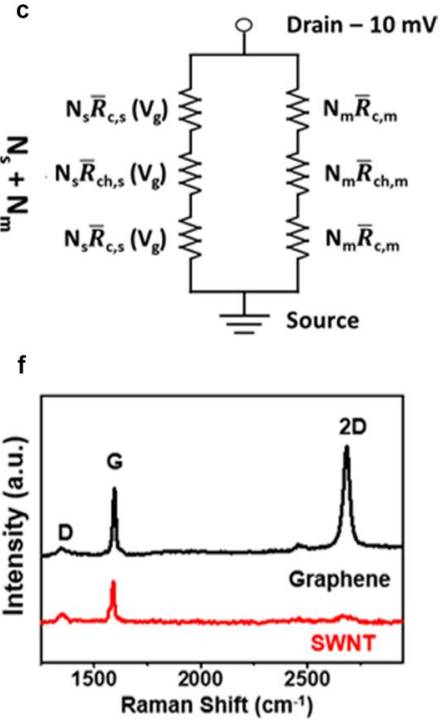

Fig. 1 Schematic illustration (a) and SEM image (b) of graphene/SWCNTs junction TLM test structure. c Equivalent circuit model for graphene/SWCNTs junction transistor. d AFM image of CVD grown SWCNTs. e Typical diameter distribution of SWCNTs measured by AFM. f The Raman spectra for graphene and SWCNT

we assume that the ratio of semiconducting SWCNTs to metallic SWCNTs is 63:37, and both the SWCNTs (channel) and graphene (electrode) were observed to have few defects in Fig. 1f. According to the chirality ratio of SWCNTs, we analyzed the characteristics using a simple equivalent circuit model, as shown in Fig. 1c. The main parameters for the model, which was used to estimate the contact resistance of between graphene/metallic SWCNTs and graphene/semiconducting SWCNTs respectively, are $\mathrm{N}_{\mathrm{m}}, \mathrm{N}_{\mathrm{s}}$ (the number of metallic and semiconducting SWCNTs, respectively), $\bar{R}_{\mathrm{c}, \mathrm{m}}, \bar{R}_{\mathrm{c}, \mathrm{s}}$ (the average contact resistance of metallic and semiconducting SWCNTs between graphene, respectively), $\bar{R}_{\mathrm{ch,m}}$ and $\bar{R}_{\mathrm{ch,s}}$ (the channel resistance of individual metallic and semiconducting SWCNTs, respectively). The control of the semiconducting tubes by the gate voltage is characterized using $10 \mathrm{mV}$ current located between the source and drain.

Figure 2a shows the $I_{d}-V_{d}$ output characteristics of the graphene/SWCNTs transistors with typical $\mathrm{L}_{\mathrm{ch}}(4,6$, 8 and $10 \mu \mathrm{m})$. The $\mathrm{L}_{\text {con }}$ and channel width were 25 and $100 \mu \mathrm{m}$, respectively. The overall drain current responses linearly with drain biases, indicating an ohmic-like contact behavior of graphene/SWCNTs junctions. From the transfer characteristics of graphene-SWCNTs transistors, measured with the source grounded, the drain voltage held at $-0.01 \mathrm{~V}$, and gate voltage swept between $\pm 30 \mathrm{~V}$ (Fig. 2b), all curves exhibit ambipolar characteristics with two separated peaks. This outcome can be explained by relative $\mathrm{p}-\mathrm{n}$ junction in the graphene/SWCNTs contact caused by the work function difference at the contact area
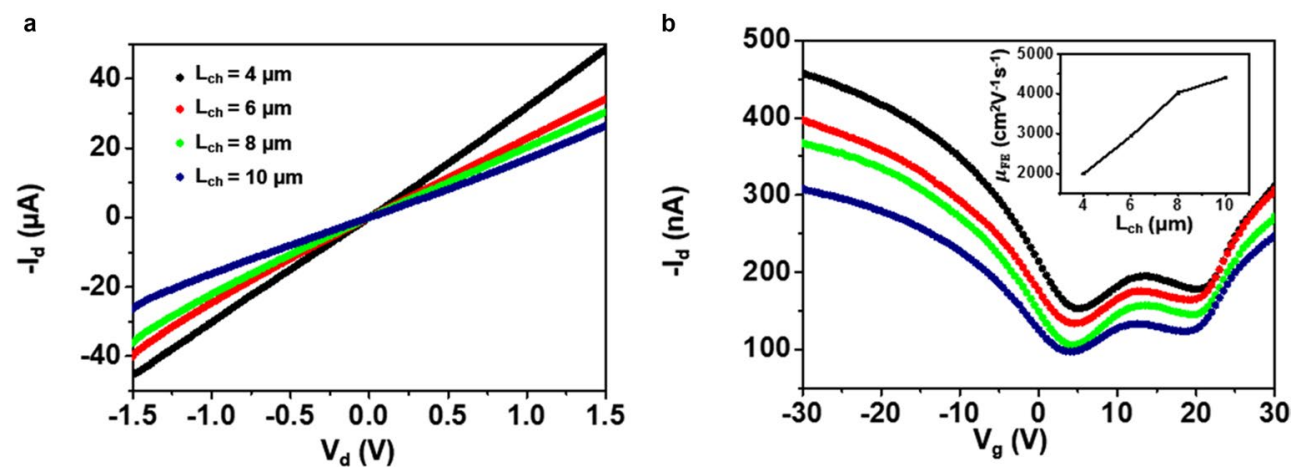

Fig. 2 a $\left.\right|_{d}-V_{d}$ characteristics $(\mathbf{b}) I_{d}-V_{d}$ transfer characteristics of graphene-SWCNTTFT with different $L_{c h}$ and a $100 \mu m$ width 
[11]. Also, the transfer curve clearly shows the p-type doing of graphene and SWCNTs induced by the external adsorbates such as $\mathrm{O}_{2}, \mathrm{CO}_{2}$ and $\mathrm{H}_{2} \mathrm{O}$. The on/off ratio ranged from $\sim 2.96$ to $\sim 3.46$, which comparable result to previous results on the metal electrode/SWCNT TFTs [3]. The inset of Fig. 2b shows calculated mobilities from devices with different $\mathrm{L}_{\mathrm{ch}} \mathrm{s}$. From the transfer curves, field effect mobilities can be calculated using the mobility calculation method. The values are as high as from 1991 to $4407 \mathrm{~cm}^{2} \mathrm{~V}^{-1} \mathrm{~s}^{-1}$. However, the monotonic decrease in mobility with decreasing $L_{c h}$ s suggests that the contact resistance plays a non-negligible role in the device operation $[12,13]$.

According to electrical device characteristics of graphene/SWCNT TFTs as shown in Fig. 2, the contact resistance for m-SWCNT and s-SWCNT are extracted from the TLM within diffusive transport regime (Fig. 3a). The 2-point total resistance $R_{\text {tot }}$ can be approximately written as the equations below:

$$
\mathrm{R}_{\mathrm{tot}}=\rho_{\mathrm{SWNT}} \mathrm{L}_{\mathrm{ch}}+2 \mathrm{R}_{\mathrm{c}}
$$

where $\rho_{\text {SWNT }}$ is the resistivity of SWCNTs and $R_{c}$ is the contact resistance between graphene and SWCNTs. The slope of the plot gives the resistivity of SWCNTs. The $\mathrm{y}$-intercept of the total resistance as a function of $\mathrm{L}_{\mathrm{ch}}$ indicates the contact resistance. Since the m-SWCNTs are, nominally, not modulated by the gate bias, we consider only s-SWCNTs in an approximate procedure that involves subtracting the minimum current which occurs at $\mathrm{V}_{\mathrm{g}}$ near $5 \mathrm{~V}$ for devices reported here, from the measured currents used in the TLM test structure. Therefore, the resistivity of SWCNTs was determined to be around $36 \mathrm{k} \Omega \mu \mathrm{m}^{-1}$ (m-SWCNT) and $56 \mathrm{k} \Omega \mu \mathrm{m}^{-1}$ (s-SWCNT), respectively. Also, the contact resistance between graphene and SWCNTs at fixed contact area ( 25 by $100 \mu \mathrm{m})$ was estimated to be around $494 \mathrm{k} \Omega(\mathrm{m}-\mathrm{SWCNT})$ and $617 \mathrm{k} \Omega(\mathrm{s}-\mathrm{SWCNT})$, respectively. As the $\mathrm{V}_{\mathrm{g}}$ in the channel is increased from -30 to $-10 \mathrm{~V}$ with steps of $4 \mathrm{~V}$, the contact resistance increases from 617 to $2316 \mathrm{k} \Omega$
(Fig. 3b). This means that the decrease of contact resistance at lower bias is related to the increase of electrostatically doped carrier density in both graphene and SWCNTs. The higher carrier density in the junction induced by negative back-gate bias enhances the carrier concentration, which leads to a narrower Schottky barrier (Fig. 3c). Meanwhile, the narrowed Schottky barrier would thus facilitate the hole injection from the graphene into the SWCNTs, leading to a lower contact resistance.

$\mathrm{L}_{\text {con }}$ scaling was studied in the same manner as $\mathrm{L}_{\mathrm{ch}}$ scaling. Figure 4a shows a plot of transfer curves of different $\mathrm{L}_{\text {con }}(5,10,15,20$, and $25 \mu \mathrm{m})$ graphene/SWCNT junction TFTs. Figure $4 \mathrm{~b}$, c shows average resistance of $\mathrm{m}$-SWCNT and s-SWCNT with different $\mathrm{L}_{\mathrm{con}} \mathrm{s}$. In the same manner as above, contact resistances were extracted and plotted on Fig. 4d [14]. The results indicate that as $\mathrm{L}_{\text {con }}$ shortened, the resistance increased. Some studied have suggested that transport between a metal and a SWCNT occurs only at the contact edge, with no $L_{\text {con }}$ dependence [15-17]. This differing tendency is caused by longer transfer length.

$$
\mathrm{L}_{\mathrm{T}}=\frac{2 \mathrm{R}_{\mathrm{c}}}{\rho_{\mathrm{ch}}}
$$

The transfer length $\left(\mathrm{L}_{\mathrm{T}}\right)$, which is the length along a contact at which the applied voltage drops to $1 / \mathrm{e}$ of its value, is extrapolated from line intercepts from the $\mathrm{x}$-axis of the TLM plot. The best fit to the data using equation below is shown by solid curve. The determined $\mathrm{L}_{\mathrm{T}}$ from fitted line are 8.74 and $9.75 \mu \mathrm{m}$ for $\mathrm{m}-\mathrm{SWCNT}$ and s-SWCNT, respectively. The experimental data agree with the fitting model. This suggest that the contact resistance depends significantly on $\mathrm{L}_{\text {con }}$ even in micrometer regime due to the long transfer length.

\section{Conclusions}

In conclusion, we have explored the contact resistance in graphene/SWCNT junctions using TLM with a simple equivalent model. The Schottky barrier between
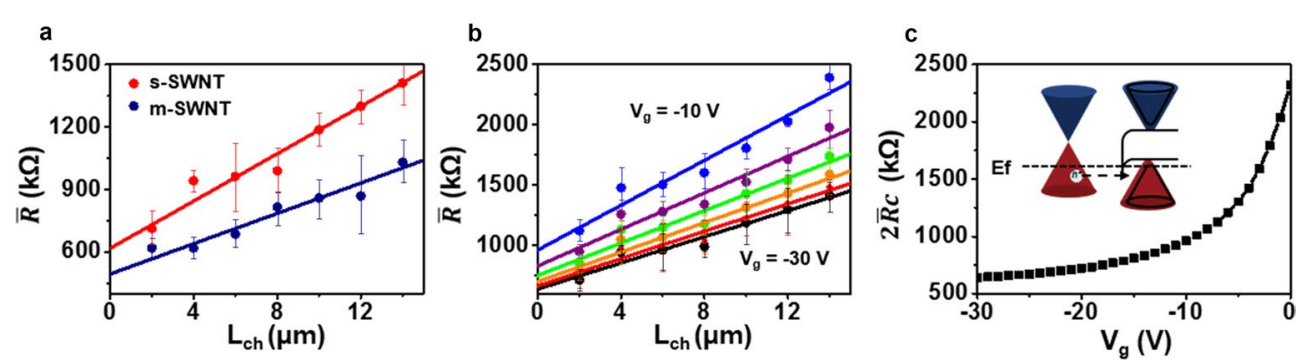

Fig. $3 L_{c h}$ scaling and extracted contact resistance. a The average resistance of the m-SWCNTs and s-SWCNT as a function of $L_{c h}$ at gate voltages of $0 \mathrm{~V}(\mathrm{~m}-\mathrm{SWCNT})$ and $-30 \mathrm{~V}(\mathrm{~s}-\mathrm{SWCNT})$, respectively. $\mathbf{b}$ The average resistance of the s-SWCNT as a function of $\mathrm{L}_{\mathrm{ch}}$, varying the back-gate from - 30 to $-10 \mathrm{~V}$ with steps of $4 \mathrm{~V}$. c The extracted contact resistance of the s-SWCNTs as a function of gate voltage 
(a)

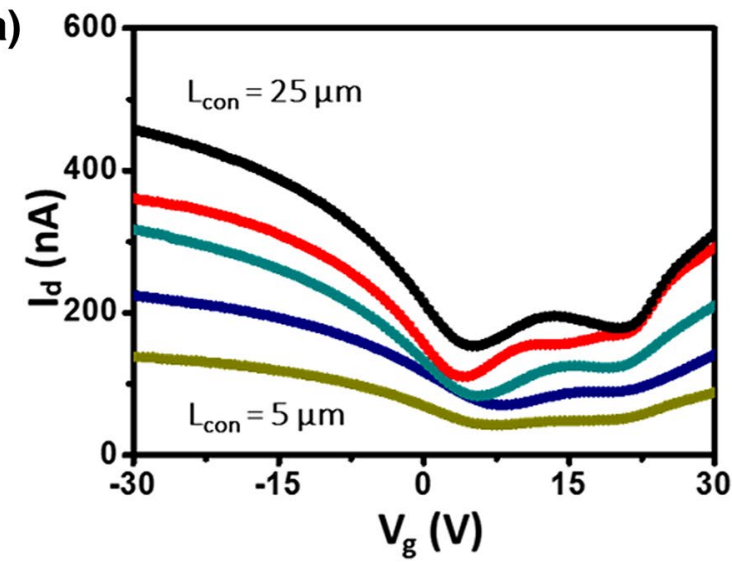

(c)

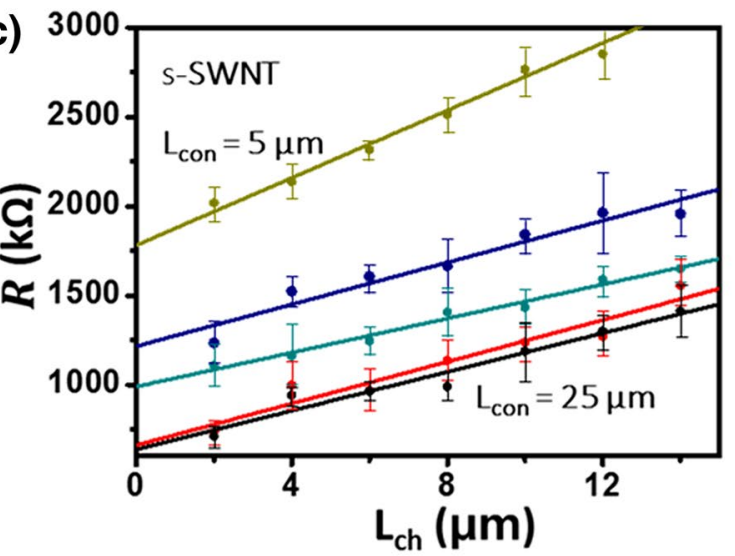

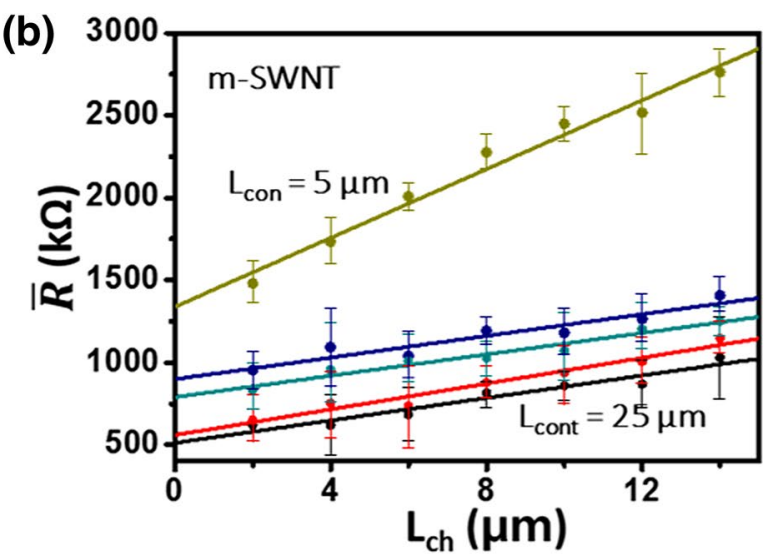

(d)

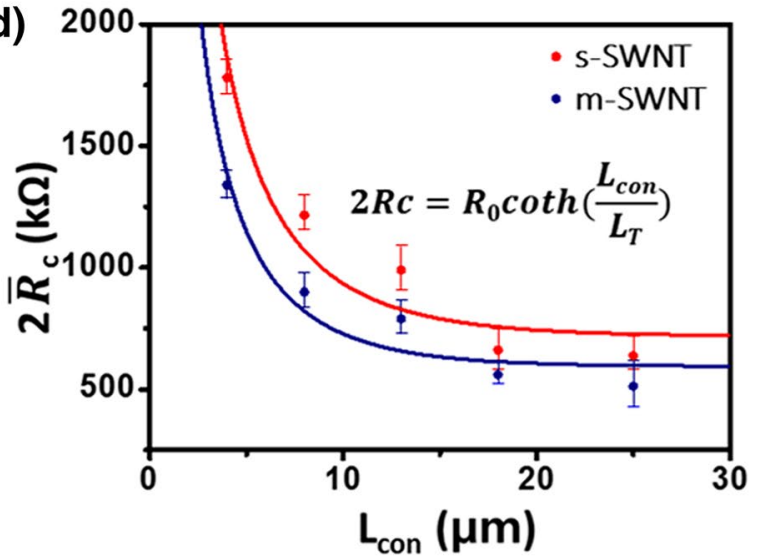

Fig. 4 a $I_{d}-V_{g}$ transfer characteristics of graphene-SWCNTTFT with different $L_{\text {con }}(5,10,15,20,25,30 \mu \mathrm{m})$ and a $10 \mu \mathrm{m}$ channel width. The average resistance of the m-SWCNTs $(\mathbf{b})$ and s-SWCNT (c) as a function of $\mathrm{L}_{c h}$ at different $\mathrm{L}_{\text {con }}$. $\mathbf{d}$ The extracted contact resistance as a function of $\mathrm{L}_{\text {con }}$ and fitting line

graphene and SWCNT leads to a p-n like junction. We investigated the contact resistance modulation of graphene/SWCNT junctions, which is controlled by backgate bias. From our measurement, the contact resistance in the junction was found to be $\sim 494$ and $\sim 617 \mathrm{k} \Omega$ in the case of m-SWCNT and s-SWCNT. We confirmed that the work function tuning of graphene and SWCNT induced by doping and controlling the $\mathrm{L}_{\text {con }}$ can alter the contact resistance in graphene/SWCNT junctions. Moreover, further development of contact engineering such as investigation of SWCNT chirality and lowering the defect density of graphene shows the potential for allcarbon based electronics.

\section{Additional file}

Additional file 1: Figure S1. Additional geometric information of graphene/SWCNT devices.

\section{Abbreviations}

SWCNT: single-walled carbon nanotube; M: metallic; S: semiconducting; $L_{\text {con }}$ : contact length; $L_{c h}$ : channel length; TLM: transfer length method; CVD: chemical vapor deposition; SEM: scanning electron microscope; AFM: atomic force microscopy; PMMA: Poly (methyl methacrylate); $\mathrm{N}_{m}, \mathrm{~N}_{s}$ : the number of metallic and semiconducting SWCNTs, respectively; $\bar{R}_{c, m}, \bar{R}_{c, s}$ : the average contact resistance of metallic and semiconducting SWCNTs between graphene, respectively; $\bar{R}_{\mathrm{ch}}, \mathrm{m}$ and $\bar{R}_{\mathrm{ch}}$, s: the channel resistance of individual metallic and semiconducting SWCNTs, respectively; $\mathrm{L}_{\mathrm{T}}$ : transfer length.

\section{Authors' contributions}

$\mathrm{JB}$, and $\mathrm{SJ}$ designed and carried out experiments and wrote the manuscript. $\mathrm{TN}, \mathrm{BK}, \mathrm{HK}, \mathrm{JL}$ prepared the samples. JB, HK, and SJ discussed the experimental results and commented on the manuscript. All authors read and approved the final manuscript.

Competing interests

The authors declare that they have no competing interests.

Ethics approval and consent to participate Not applicable.

\section{Funding}

This work was supported by Future Planning as Global Frontier (CASE2013M3A6A5073173), and nanomaterial technology development program through the National Research Foundation of Korea (NRF) funded by the 
Ministry of Science. This work was also supported by the National Research Foundation of Korea (NRF) grant funded by the Ministry of Science, ICT \& Future Planning (2015R1A2A1A15053051) and the BK21 plus program through the National Research Foundation (NRF) funded by the Ministry of Education of Korea.

\section{Publisher's Note}

Springer Nature remains neutral with regard to jurisdictional claims in published maps and institutional affiliations.

Received: 4 October 2017 Accepted: 27 November 2017

Published online: 20 December 2017

\section{References}

1. A.K. Geim, K.S. Novoselov, Nat. Mater. 6, 183-191 (2007)

2. A.K. Geim, Science 324, 1530-1534 (2009)

3. S.J. Kang, C. Kocabas, T. Ozel, M. Shim, N. Pimparkar, M.A. Alam, Nat. Nanotechnol. 2, 230-236 (2007)

4. E. Artukovic, M. Kaempgen, D.S. Hecht, S. Roth, G. GrUner, Nano Lett. 5, 757-760 (2005)

5. D.M. Sun, C. Liu, W.C. Ren, H.M. Cheng, Small 9, 1188-1205 (2013)
6. T. Pei, H.T. Xu, Z.Y. Zhang, Z.X. Wang, Y. Liu, Y. Li, Appl. Phys. Lett. 99, $113102(2011)$

7. M. Seo, H. Kim, Y.H. Kim, J. Na, B.J. Lee, J.J. Kim, Appl. Phys. Lett. 107, 033103 (2015)

8. Y. Kim, J. Lee, M.S. Yeom, J.W. Shin, H. Kim, Y. Cui, J.W. Kysar, J. Hone, Y. Jung, S. Jeon, S.M. Han, Nat. Commun. 4, 2114 (2013)

9. J. Lee, B.H. Kim, S.J. Kang, K.T. Hyun, S.H. Lee, S. Jeon, MRS Commun. 2 79-83 (2012)

10. W. Kim, A. Javey, R. Tu, J. Cao, Q. Wang, H.J. Dai, Appl. Phys. Lett. 87 $173101(2005)$

11. K. Yan, D. Wu, H.L. Peng, L. Jin, Q. Fu, X.H. Bao, Z.F. Liu, Nat. Commun. 3 1280 (2012)

12. X.J. Zhou, J.Y. Park, S.M. Huang, J. Liu, P.L. McEuen, Phys. Rev. Lett. 95 146805 (2005)

13. Z.H. Chen, J. Appenzeller, J. Knoch, Y.M. Lin, P. Avouris, Nano Lett. 5 , 1497-1502 (2005)

14. D.K. Schroder, Semiconductor Materials and Device Characterization, 3rd edn. (Wiley, Hoboken, 2006), pp. 127-184

15. X.F. Song, X.B. Han, Q. Fu, J. Xu, N. Wang, D.P. Yu, Nanotechnology 20 $195202(2009)$

16. D. Mann, A. Javey, J. Kong, Q. Wang, H.J. Dai, Nano Lett. 3, 1541-1544 (2003)

17. Y. Nosho, Y. Ohno, S. Kishimoto, T. Mizutani, Jpn. J. Appl. Phys. 46, L474L476 (2007)

\section{Submit your manuscript to a SpringerOpen ${ }^{\circ}$ journal and benefit from:}

- Convenient online submission

- Rigorous peer review

- Open access: articles freely available online

- High visibility within the field

- Retaining the copyright to your article

Submit your next manuscript at $\boldsymbol{\nabla}$ springeropen.com 